\title{
El autocuidado de los adolescentes que están en diálisis
}

\author{
Self-care for teenagers on dialysis
}

Liliana Cristina Morales-Viana ${ }^{1 *}$ orcid.org/0000-0002-3805-4958

1 Facultad de Salud, Universidad del Valle. Cali, Colombia.

Fecha de recepción: Agosto 15 - $2017 \quad$ Fecha de revisión: Junio 26-2018 Fecha de aceptación: Diciembre 11 - 2018

Morales-Viana LC. El autocuidado de los adolescentes que están en diálisis. Univ. Salud. 2019;21(1):88-99. DOI: http://dx.doi.org/10.22267/rus.192101.142

\section{Resumen}

Introducción: El autocuidado de los adolescentes con Insuficiencia Renal Crónica Terminal (IRCT) que están en diálisis, ha sido poco estudiada, pese a que constituye un mecanismo importante para el cuidado de la salud Objetivo: Identificar la evidencia científica sobre el autocuidado de adolescentes con IRCT en diálisis. Materiales y métodos: Se consultó las bases de datos Biomed Central-Open Acces, E-Books Colletion EBSCO, EBSCO, Global Health, ISI Web of Science, OVID, PubMed Central, SciELO, ScienceDirect, SCOPUS y Springer Link, utilizando las palabras clave: autocuidado, adolescentes, enfermedades crónicas no transmisibles, insuficiencia renal crónica terminal, adherencia, adherencia farmacológica, adherencia al tratamiento, en español e inglés. Resultados: De 452 artículos consultados se seleccionaron 84 que cumplían los criterios de inclusión y se construyó cuatro capítulos de análisis: adherencia al tratamiento, restricción en la ingesta de líquidos, intervenciones orientadas a fortalecer la adherencia terapéutica y aproximaciones cualitativas al autocuidado de los adolescentes con IRCT. Conclusiones: El autocuidado de los adolescentes con IRCT en diálisis, ha sido abordado principalmente desde un enfoque cuantitativo; múltiples factores interfieren en la adherencia al tratamiento, por tanto, se evidencia la necesidad de nuevos abordajes para hacer frente al desafío del autocuidado tanto en la práctica como en la investigación.

Palabras clave: Autocuidado; adolescentes; insuficiencia renal crónica; adherencia al tratamiento. (Fuente: DeCS, Bireme).

\begin{abstract}
Introduction: Self-care for adolescents with chronic end-stage renal failure who are on dialysis has been understudied; although, it constitutes an important mechanism for health care. Objective: To identify the scientific evidence on the self-care of adolescents with chronic end-stage renal failure on dialysis. Materials and methods: The Biomed Central-Open Acces, E-books collation EBSCO, EBSCO, Global Health, ISI Web of Science, OVID, PubMed Central, SciELO, ScienceDirect, SCOPUS and Springer Link databases were consulted by using the keywords: self-care, adolescents, chronic noncommunicable diseases, chronic end-stage renal failure, adherence, pharmacological adherence, adherence to treatment, in Spanish and English. Results: From 452 articles consulted, 84 were selected because they met the inclusion criteria and four chapters of analysis were built: adherence to treatment, restriction in the intake of fluids, interventions aimed at strengthening therapeutic adherence and qualitative approximations to the self-care of adolescents with chronic end-stage renal failure. Conclusions: Self-care of adolescents with chronic end-stage renal failure on dialysis has been addressed mainly from a quantitative approach. Multiple factors interfere with adherence to treatment; therefore, the need for new approaches to cope with the challenge of self-care both in practice and in research is evident.
\end{abstract}

Palabras clave: Self-care; adolescent; renal insufficiency, chronic; treatment adherence. (Fuente: DeCS, Bireme).

\footnotetext{
*Autor de correspondencia

Liliana Cristina Morales Viana

e-mail: liliana.morales@correounivalle.edu.co
} 


\section{Introducción}

El autocuidado ha sido descrito como un movimiento, un concepto, un marco, un modelo, una teoría, un proceso o un fenómeno. Las diferentes perspectivas de autocuidado, no han permitido una definición única, y ampliamente aceptada en la literatura científica. Al respecto, Levin que es considerado como el "padre" del autocuidado, sugiere que lograr una definición común "sólo puede ser posible a costa de perder la precisión requerida para una investigación significativa"(1). No obstante, Godfrey et al.(2), realizaron el análisis de 139 definiciones, concluyendo que el autocuidado es la capacidad de la persona en estado saludable, de enfermedad $o$ de discapacidad, para comprometerse con actividades de promoción de la salud física, mental y emocional, mantener la vida y prevenir las complicaciones, ya sea por cuenta propia o en colaboración con su familia y los servicios de salud(2).

Debido al gran potencial positivo que tiene el autocuidado sobre la forma de vivir de las personas, éste constituye una estrategia importante para la protección de la salud y la prevención de la enfermedad(3). La Organización Mundial de la Salud (OMS), en los objetivos de las "Políticas de salud para todos en el siglo XXI", concede una elevada importancia a la participación activa de los ciudadanos en el cuidado de la salud; considera el fomento del autocuidado como uno de los principales ejes y lo describe como las actividades que los individuos, las familias y las comunidades emprenden con la intención de mantener la salud, prevenir o controlar la enfermedad y restaurar la salud(4).

El autocuidado ha demostrado ser una práctica invaluable pues se trata de una forma efectiva y segura de prevenir la aparición de una importante cantidad de problemas de salud, pero especialmente, ser efectivo en el fortalecimiento de la salud de las personas con enfermedades crónicas no transmisibles (ECNT), ya que constituye una de las prioridades para el mantenimiento de su salud, disminución de complicaciones y discapacidades(5). Por su parte, en Colombia y el mundo, las ECNT se han convertido en un fenómeno de salud de interés, dado que forman parte de las primeras causas de morbilidad $\mathrm{y}$ mortalidad en los perfiles epidemiológicos(6). A nivel mundial, las principales ECNT son las enfermedades cardiovasculares, el cáncer, las enfermedades respiratorias crónicas y la diabetes(7). Las ECNT causan impacto en la vida de las personas, sus familias, sus cuidadores, la comunidad, el sistema de salud y la sociedad en general( ${ }^{(8)}$. Cuando ocurren durante la adolescencia, el impacto es mayor debido a que la enfermedad interfiere en el proceso de crecimiento y desarrollo del joven( ${ }^{(9)}$; además representan el 20,5\% del total de Años de Vida Potencialmente Perdidos (AVPP)(10); el fallecimiento prematuro de los adolescentes genera gran impacto en la productividad económica de los países(11).

En las últimas décadas se han producido importantes cambios en la epidemiología de las ECNT en la adolescencia. Estudios realizados en Estados Unidos e Inglaterra muestran que del $5 \%$ al 30\% de los adolescentes padecen al menos una enfermedad crónica no trasmisible(12). En el contexto colombiano, durante el periodo comprendido entre 2009 y 2014, las ECNT en esta población fueron la primera causa de las intervenciones en salud correspondientes al $56,8 \%$ de la demanda, con una razón de 3,84 atenciones por persona ${ }^{(13)}$. Entre las principales ECNT en este grupo se encuentran las enfermedades renales, cardiovasculares y endocrinológicas(14-18). Las enfermedades renales, particularmente la Insuficiencia Renal Crónica Terminal (IRCT) es uno de los padecimientos crónicos más complejos en los adolescentes, con incidencia y prevalencia creciente, es considerada como enfermedad catastrófica por la pérdida progresiva e irreversible de la función renal(19), por tanto, el paciente depende en forma permanente de un tratamiento sustitutivo que puede ser hemodiálisis, diálisis peritoneal o trasplante(20).

La terapia dialítica y el trasplante renal son dos aspectos clave que contribuyen a la alta complejidad de la IRCT, puesto que los jóvenes que se exponen a estas dos acciones terapéuticas 
tienen una tasa de mortalidad de entre 30 a 150 veces comparada con la de la población pediátrica general y una calidad de vida severamente deteriorada(21). En 1988 Estados Unidos reportó una prevalencia de esta enfermedad del $13 \%$ en la población joven, mientras que para el 2014 la fue de $16 \%$ (22). Los registros europeos muestran incidencias alrededor de 10-12 pacientes por millón de población pediátrica (ppmp) y prevalencias alrededor de 59-74 ppmp(23). La incidencia para América Latina tiene un amplio rango que va de 2,8 a 15,8 casos nuevos anuales por millón de habitantes menores de 15 años, esto debido a condiciones como la distribución geográfica de la población y la situación socioeconómica de cada país(23). Para Colombia, la prevalencia es $66,8 \mathrm{x}$ cada 100.000 habitantes, siendo más alta en hombres que en mujeres. Los departamentos con mayores prevalencias son: Valle del Cauca, seguido por Sucre, Huila, Quindío y Bolívar. Los datos anteriores son para todas las edades debido a que no hay un registro exclusivo para adolescentes $(24,25)$.

El tránsito por la adolescencia representa un reto en sí mismo, si además se le añaden las exigencias de una ECNT como la IRCT, los jóvenes tienen que equilibrar las tareas habituales del desarrollo con las tareas adaptativas presentadas por su condición de salud. La IRCT produce en los jóvenes síntomas como fatiga y dolor permanentes, sufren de deterioro cognitivo y por consiguiente tienen peores resultados educativos, vocacionales y psicosociales en comparación con sus pares que enfrentan otras patologías crónicas, y que sus pares sanos(26). Igualmente tienen mayores problemas de ajuste psicosocial que sus pares, por aspectos como el menor desarrollo puberal, mayor pérdida de clases y menor capacidad de "conectarse" a una red social, hechos que pueden llevarlos a la depresión y al intento suicida(27).

Mejorar el autocuidado de los adolescentes con IRCT, se considera un elemento importante en las estrategias para reducir la enorme y creciente carga de la enfermedad y el costo que genera(28). Además, el fortalecimiento del autocuidado en estos jóvenes puede permitirles convivir con su situación de salud de una manera menos traumática. Por lo anterior, los sistemas de salud a nivel mundial hacen cada vez más hincapié en el autocuidado como un componente importante de los modelos integrales de salud(29,30). No obstante, los esfuerzos dedicados a generar desarrollos con este fin son aún insuficientes, se requiere, entre otros aspectos, de modelos y programas de atención adecuados al contexto de los adolescentes ${ }^{(31)}$.

Teniendo en cuenta la importancia del autocuidado en los jóvenes con IRCT, se realizó una revisión crítica de literatura sobre este tema; se recuperó artículos científicos publicados entre los años 2007 a 2017, la búsqueda mostró cuatro aspectos que constituyen las categorías que están descritas en la sección de resultados y discusión, el primer tema se relaciona con la adherencia al tratamiento, el segundo de estudios sobre la restricción en la ingesta de líquidos, el tercero corresponde a estudios sobre las intervenciones orientadas a fortalecer la adherencia terapéutica y el cuarto son las aproximaciones cualitativas al autocuidado de los adolescentes con IRCT. Es importante destacar que la mayoría de los estudios sobre el autocuidado de los adolescentes con IRCT han sido realizados con metodologías cuantitativas, lo que resulta insuficiente para conocer un fenómeno subjetivo tan complejo.

Conocer sobre el autocuidado en los adolescentes con enfermedades como la IRCT, es útil para que los profesionales de la salud, particularmente las enfermeras, fundamenten sus intervenciones en la evidencia que proporciona la literatura científica, de igual manera permite a los investigadores interesados en el tema, identificar vacíos en el conocimiento para guiar sus estudios. Los resultados de este tipo de investigaciones deben ayudar a unos y otros a comprender el autocuidado como parte fundamental de la vida de los jóvenes.

Los estudios sobre el autocuidado en los adolescentes con IRCT en diálisis, considerados para este artículo han sido insuficientes para comprender el autocuidado desde una perspectiva integral, se requiere fortalecer el 
abordaje cualitativo que permita conocer el mundo de los jóvenes, cómo viven y construyen en su cotidianidad el cuidado de sí mismos.

\section{Materiales y métodos}

Para la presente revisión se recuperó artículos que cumplieron con los siguientes criterios de inclusión: (1) haber sido publicados entre el 2007 y el 2017, (2) en las bases de datos: Biomed Central-Open Acces, E-Books Colletion EBSCO, EBSCO, Global Health, ISI Web of Science, OVID, PubMed Central, SCIELO, Science Direct, SCOPUS, Springer Link. (3) en idiomas español e inglés. Los términos utilizados para la búsqueda en español fueron: autocuidado, adolescentes, enfermedades crónicas no transmisibles, insuficiencia renal crónica, adherencia, adherencia farmacológica, adherencia al tratamiento; en inglés fueron: self-care, adolescents, chronic noncommunicable diseases, chronic renal failure, adherence, pharmacological adherence, adherence to treatment. Se utilizaron los conectores boleanos AND, OR y NOT.

El contenido de los artículos se organizó en una ficha técnica elaborada para tal fin, la cual contenía los siguientes ítems: referencia bibliográfica, objetivos del estudio, metodología, resultados, principales conclusiones, recomendaciones e implicaciones. Posteriormente, se hizo la evaluación de los artículos para determinar su calidad y cumplimiento de los criterios de inclusión, para finalmente hacer el análisis e interpretación de los artículos seleccionados.

\section{Resultados}

Se recuperaron 452 artículos relacionados con el autocuidado de los adolescentes con IRCT, de los cuales 84 cumplieron los criterios de inclusión, (Figura 1).

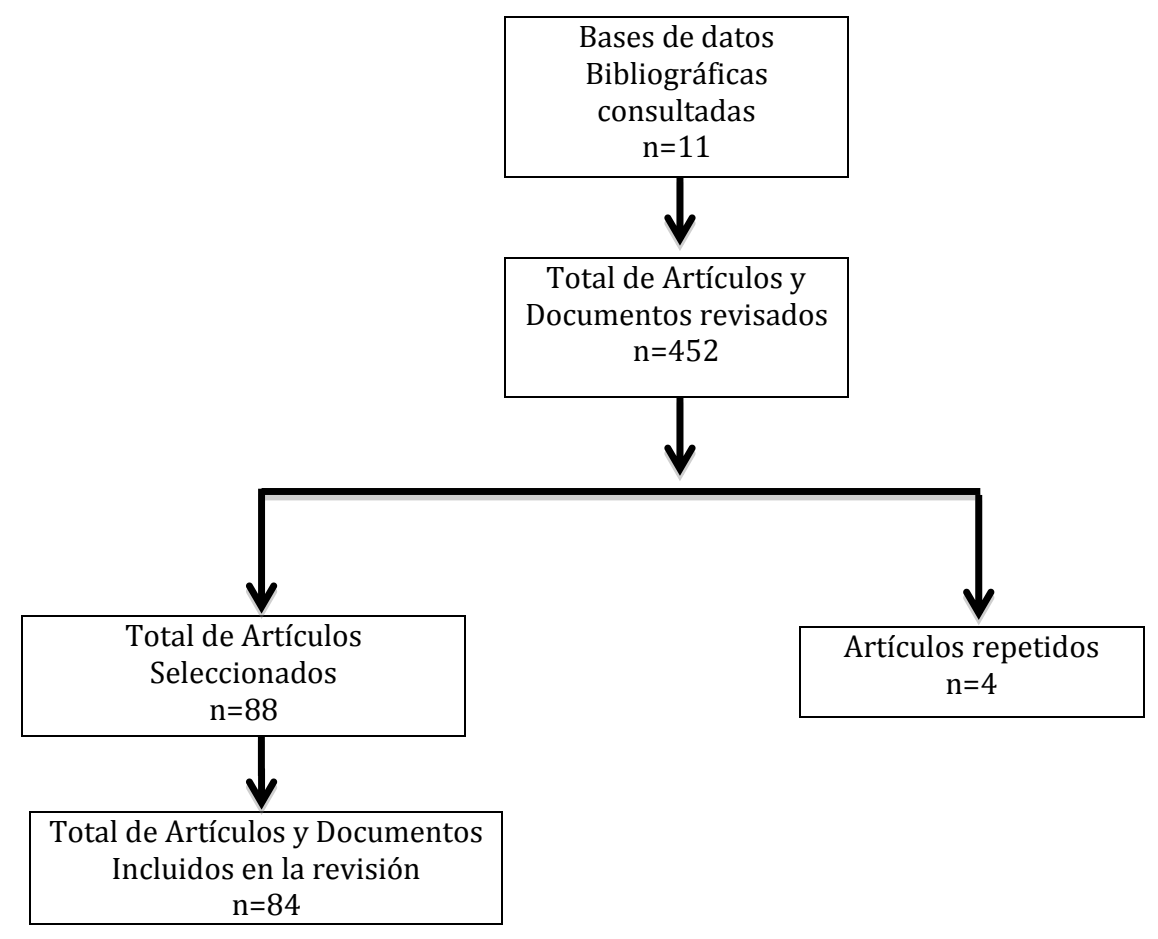

Figura 1. Selección de documentos sobre el autocuidado de los adolescentes en diálisis 
De la revisión de los artículos se identificaron 4 categorías.

\section{Adherencia al tratamiento}

El autocuidado en los adolescentes con IRCT en diálisis, al igual que el autocuidado en los adolescentes con otras ECNT se ha estudiado esencialmente como la adherencia al tratamiento o adherencia terapéutica. La OMS define la adherencia terapéutica como el grado en que el comportamiento de una persona (tomar el medicamento, seguir un régimen alimentario y ejecutar cambios del modo de vida), se corresponde con las recomendaciones acordadas por un prestador de asistencia sanitaria(32). DiMatteo y $\operatorname{DiNicola}^{(33)}$ definen la adherencia como "una implicación activa y voluntaria del paciente en el curso de un comportamiento aceptado de mutuo acuerdo con su médico, con el fin de producir un resultado terapéutico deseado". Haynes, 1979 la definió como "la medida en que el comportamiento de una persona coincide con el asesoramiento médico o de salud", siendo ésta la definición más aceptada(34). Existe una fuerte evidencia de que los adolescentes con diversas condiciones crónicas de salud tienen mayor dificultad para adherirse a su régimen recomendado, por tal motivo la adherencia a los diferentes tratamientos resulta más desafiante en esta etapa de la vida(35,36).

Se han discutido varias cuestiones relacionadas con la adherencia terapéutica durante la adolescencia; en primer lugar aspectos propios del desarrollo de esta etapa, como la independencia y la individuación, pueden llevar al joven a tener una adherencia menos óptima a un régimen diario exigente. Otros eventos del desarrollo tales como socializar con sus compañeros o trabajar por primera vez, pueden interferir con la programación de las actividades relacionadas con el régimen terapéutico. Además, la naturaleza permanente y severa de la enfermedad puede ser más evidente y desalentadora durante la adolescencia, la comprensión de que la enfermedad continuará incluso cuando se es totalmente adherente con el tratamiento puede ser decepcionante y dar lugar a la no adherencia(37).
Varios autores(38-40) han estudiado las tasas de adherencia a las terapias farmacológicas en jóvenes con IRCT en diálisis. La conclusión general de estos estudios es que la deficiente adherencia a los fármacos es frecuente entre estos pacientes. No obstante, su medición es compleja pues se desconoce el porcentaje mínimo de adherencia necesario para obtener los beneficios completos de un fármaco, arbitrariamente se ha fijado en el $80 \%{ }^{(38)}$. Adicionalmente, los métodos utilizados para medir la adherencia al fármaco no son muy fiables y tienden a sobrestimarla, puesto que las metodologías utilizadas como cuestionarios, entrevistas, recuentos periódicos de las dosis devueltas y no recibidas de los pacientes, están sujetas a muchas incertidumbres y a fácil manipulación por parte de los mismos.

Actualmente, el monitoreo electrónico de la adherencia al fármaco es probablemente el método más utilizado para evaluar el comportamiento de los pacientes hacia el tratamiento farmacológico,(38) pero este método tampoco es confiable. Adicionalmente, los jóvenes con IRCT pertenecen al grupo de personas con una de las cargas más altas de la ingesta diaria de pastillas con hasta 20 pastillas. Por consiguiente, el número de fármacos es un determinante importante de la adherencia en el largo plazo en personas con ECNT(38).

En 2010, ante la escasez de instrumentos para medir la adherencia al tratamiento farmacológico en la población pediátrica con enfermedad renal, el Departamento de Nefrología Pediátrica del Centro de Ciencias de la Salud de la Universidad de Texas desarrolló el Cuestionario de Adherencia a los Medicamentos de Niños y Adolescentes (CAAMQ). El instrumento fue construido para identificar los factores que pueden influir en la no adherencia de los pacientes a los medicamentos. Vasylyeva et al.(39), utilizaron este instrumento con niños y adolescentes de 10 a 21 años de edad y reportaron como el principal hallazgo, que los adolescentes tenían problemas para recordar tomar sus medicamentos, principalmente durante los fines de semana; los jóvenes preferían tomar sus medicamentos en lugares 
que aseguraran la privacidad; la Prednisona era el "más "odiado de todos los medicamentos. Las mujeres eran más reactivas y reservadas que los hombres en cuanto a dar a conocer a los compañeros su enfermedad, el tratamiento y los horarios de sus medicamentos. Ante estos hallazgos los autores recomiendan desarrollar y validar recordatorios creativos e individualizados(39). Los autores también identificaron sentimientos de tristeza, angustia y la importancia de fuertes sistemas de apoyo familiar para estos jóvenes.

Otro aspecto de gran interés y preocupación para los investigadores son las barreras para la no adherencia al tratamiento y particularmente la no adherencia a la hemodiálisis. Los personas (entre las que se encuentran los adolescentes) con IRCT se consideran no adherentes al tratamiento dialítico, si omiten una o más sesiones de diálisis al mes, acortan una o más sesiones por más de 10 minutos al mes, tienen un nivel de potasio sérico $>6,0 \mathrm{mEq} / \mathrm{L}$, un nivel de fosfato sérico $>7,5 \mathrm{mg} / \mathrm{dL}$ ó $>2,4 \mathrm{mmol} / \mathrm{L}$, o ganancia de peso interdiálisis $>5,7 \%$ del peso corporal(40). Entre los factores asociados a la no adherencia a la hemodiálisis se encuentra principalmente la depresión y la ansiedad; también se describe las dificultades para el transporte, el mal clima, las vacaciones, el dolor y los trastornos gastrointestinales. Los estudios indican que los adolescentes son los que más frecuentemente presentan inasistencia a la diálisis. Los anteriores factores también están asociados al exceso de hospitalizaciones, visitas a las salas de emergencias y admisiones a la unidad de cuidados intensivos, y a mayor mortalidad, siendo la depresión el factor más investigado(41-43).

La depresión es la condición psicológica y la comorbilidad psiquiátrica más común entre los adolescentes que reciben hemodiálisis, con una prevalencia de aproximadamente del 20\% a $30 \%(44,45)$. No obstante, el sub diagnóstico de la depresión es un reto en estos jóvenes debido principalmente a que muchos signos de depresión como la anorexia, la fatiga, la irritabilidad, la disminución del deseo sexual y el trastorno del patrón de sueño son similares a los signos del estado urémico. Por lo tanto, la verdadera epidemiología de la depresión en jóvenes con IRCT es aún desconocida(45). Varios estudios han identificado asociación entre la depresión y la no adherencia al régimen de hemodiálisis(46), por tal motivo la depresión en jóvenes con IRCT ha merecido mucha atención científica pues además se ha asociado con una menor calidad de vida, mayor comorbilidad y disminución de la supervivencia(47).

\section{Las restricciones en la ingesta de líquidos}

Las restricciones en la ingesta de líquidos es otro aspecto que presenta la literatura científica como un componente importante del autocuidado en los jóvenes con IRCT en diálisis; la ganancia inter-dialítica de peso es el indicador utilizado para conocer y controlar este aspecto. Si bien la restricción de los líquidos permite paliar adecuadamente las alteraciones hidroelectrolíticas producidas por la IRCT, también constituye otro factor que interfiere en la adherencia al tratamiento. Un estudio etnográfico realizado por Waters ${ }^{(48)}$ encontró que la restricción de líquidos es un aspecto especialmente estresante para los jóvenes, debido a que la cantidad de líquidos permitida se agota con la ingesta de las comidas y la toma de los medicamentos, lo cual hace que los jóvenes no cumplan con dicha recomendación. Al mostrar cómo los niños respondieron a su angustiosa experiencia de sed, los resultados indican que este aspecto necesita investigación urgente. Además, la complejidad de los regímenes de medicamentos sugiere que esta área requiere investigación desde las perspectivas de los niños y jóvenes. La literatura se centra en la toma de tabletas como un problema relacionado con el comportamiento de los jóvenes pero este estudio revela que el problema radica en la restricción de líquidos, las características de las tabletas y no en los jóvenes(48).

\section{Intervenciones para fortalecer la adherencia} Otro tópico que ha sido estudiado con relación al autocuidado de los adolescentes con IRCT en diálisis son las diferentes intervenciones orientadas a fortalecer la adherencia al tratamiento debido a que existe incertidumbre 
sobre la mejor manera de optimizar la adherencia al tratamiento en este grupo de edad(36). Los ensayos aleatorizados constituyen la principal metodología utilizada para este fin; Cukor et al.(47), teniendo en cuenta que la depresión en los pacientes con IRCT constituye una de las principales barreras para la adherencia al tratamiento, realizaron un ensayo aleatorizado con pacientes que asistían a dos centros de diálisis en la ciudad de Nueva York. La intervención consistió en una terapia cognitivoconductual individual administrada durante las sesiones de diálisis por 3 meses. El grupo intervenido logró reducciones significativamente mayores en el Inventario de Depresión de Beck II (auto-reportado, $\mathrm{p}=0,03$ ) y la Escala de Depresión de Hamilton (informado por el médico $\mathrm{p}=0,001$ ).

En coherencia con los anteriores autores, Keppeke et al.(36), en su revisión sistemática de ensayos controlados sobre intervenciones para mejorar la adherencia a la medicación en adolescentes, sugieren que las intervenciones educativas por sí solas son insuficientes para promover la adherencia en niños y adolescentes $\mathrm{y}$, que la incorporación de un componente conductual a las intervenciones de adherencia puede aumentar la eficacia. Así mismo, un metaanálisis realizado por Pai y McGrady(49) mostró que las acciones tuvieron pocos efectos en la post-intervención y seguimiento. Estos autores concluyeron que estos reflejan las limitaciones metodológicas de los estudios incluidos y la necesidad de reexaminar el suministro y los mecanismos de las intervenciones promotoras de la adherencia.

\section{Estudios cualitativos sobre el autocuidado}

Hay importantes avances en materia de aproximaciones cualitativas para conocer las creencias, experiencias, sentimientos, costumbres entre otros fenómenos relacionados con la salud y enfermedad en las personas con ECNT. Los estudios cualitativos se han realizado en jóvenes con otras enfermedades principalmente diabetes(50,51), asma y cáncer. Visekruna et al.(50), realizaron un estudio utilizando la fenomenología descriptiva para descubrir las experiencias de autocuidado en nueve jóvenes que vivían con diabetes T1. Los hallazgos del estudio revelaron cinco temas: 1) eludir el control; 2) dualismo de la tecnología; 3) pronosticar y mantener rutinas; 4) lidiar con los "altibajos" y, 5) la interacción con el equipo de salud. La esencia de la experiencia para las jóvenes giraba en torno a tratar de lograr "estar en equilibrio", que requería la capacidad de apropiarse de la diabetes y el autocuidado $\mathrm{y}$, mantener la cohesión con otros aspectos de la vida diaria. Para estas jóvenes, el autocuidado abarcaba el deseo y la necesidad de estar en equilibrio con su vida y los niveles de azúcar en sangre. Uno de los sentimientos más comunes entre las jóvenes fue el miedo a no lograr un buen control y padecer complicaciones a largo plazo como la neuropatía, la nefropatía y la ceguera, lo cual constituyó la principal motivación para el autocuidado. Las jóvenes describieron que su autocuidado giraba alrededor de cinco aspectos: el monitoreo rutinario de la glucosa en la sangre, los cálculos exactos de los carbohidratos y las dosis de insulina, el comer consistentemente y la participación en la actividad física. Si bien las jóvenes comprendieron la importancia de incorporar el ejercicio en su estilo de vida, a menudo les resultó difícil, así como frustrante al manejar las fluctuaciones en los niveles de glucosa en sangre en esos momentos. El cálculo de las dosis de insulina y la ingesta de carbohidratos antes de la actividad física eran necesarios. Ellas describieron un proceso de cálculos de "ensayo y error" como la estrategia para determinar la ingesta apropiada de insulina y carbohidratos para esa actividad. Pese al reconocimiento de la importancia de sostener los comportamientos y actividades de autocuidado, las demandas de la vida cotidiana interfirieron con su compromiso. Por otra, parte expresaron que el autocuidado de una enfermedad como la diabetes no es un proceso lineal sino una experiencia iterativa de altos y bajos ${ }^{(50)}$.

Spencer et al.(51), en una revisión sistemática de estudios cualitativos con adolescentes con diabetes $\mathrm{T} 1$ encontraron que las relaciones sociales son un factor clave en el autocuidado de esta enfermedad. Por un lado, los adolescentes se enfrentan a malabares con las diferentes 
demandas de su diabetes dentro de la familia, la escuela, los compañeros y las instituciones de salud, y por otro lado, las habilidades de los adolescentes para ser independientes en el autocuidado de la diabetes se insertan en las relaciones con los padres, compañeros, profesionales de la salud y maestros. Si bien los parámetros clínicos, incluidos los valores diarios de glucosa en sangre y A1c, pueden ser vitales para identificar y evaluar posibles complicaciones, los elementos de la individualidad que afectan las preferencias, metas, desafíos, actitudes y estilos de vida exigidos por el tratamiento no pueden ser evadidos. En el contexto de la relación pacienteprofesional, las personas esperan apoyo más allá de los números $(50,51)$.

En los últimos años se ha observado un aumento exponencial de las personas con condiciones de salud a largo plazo utilizando internet para obtener información y apoyo(52). Allen et al.(53), realizaron una revisión sistemática de estudios cualitativos para identificar cómo la información y la comunicación entre las comunidades en línea de personas con ECNT, como la IRCT, pueden ayudar al autocuidado en su vida cotidiana. Se determinaron cuatro mecanismos para el apoyo en el autocuidado en las comunidades de personas: 1) el conocimiento colectivo y la identificación a través de la experiencia vivida; 2) apoyo, información y compromiso a través de relaciones fácilmente accesibles; 3) la sociabilidad que se extiende más allá de la enfermedad y 4) la desinhibición en línea como facilitador en la negociación del apoyo del autocuidado. Las comunidades en línea pueden permitir a los miembros comunicarse con sus compañeros para obtener consejos útiles sobre la enfermedad, desarrollar conocimientos sobre las prácticas de tratamiento diario a través de ensayo y error, dándoles valiosos conocimientos sobre las habilidades cotidianas de autocuidado para equilibrarlas en su vida diaria. Las personas aprendieron de las prácticas de autocuidado de otros, observando sus estrategias, otras nuevas y más eficientes; a partir de esto, fueron capaces de seleccionar el enfoque que mejor se ajustaba a sus necesidades de autocuidado.
Las comunidades en línea facilitan el empoderamiento de las personas ofreciendo a los miembros el derecho de explorar el autocuidado de su condición de salud en el contexto de la vida cotidiana. Esta perspectiva de empoderamiento de los pacientes permite reconocer que el conocimiento colectivo creado a través de la experiencia vivida es más útil en el autocuidado de las personas con ECNT en la cotidianidad que el conocimiento médico(51).

Wells, et al.(54), estudiaron las vivencias de adolescentes con el tratamiento farmacológico, dialítico y nutricional. Los jóvenes describieron el tratamiento como desafiante y de impacto significativo en las relaciones y las rutinas diarias. Pese al gran impacto y sufrimiento que generaba el tratamiento, ellos no tenían otra opción que priorizar su salud e integrar los tratamientos en su vida, dado que su condición de salud la amenazaba; reconocieron que tomar medicamentos, asistir o realizarse la diálisis y cumplir con las restricciones dietéticas era increíblemente exigente e impactante. Varios factores intervenían en la adherencia al tratamiento; por ejemplo, tomar la medicación a menudo dependía de si encajaba en sus rutinas diarias. Todos los jóvenes plantearon conflictos entre la aceptación de los pares y las demandas de la enfermedad y el tratamiento. Un sentido de identidad y no ser etiquetados como "enfermos" era un tema importante para la mayoría de ellos. Estos hallazgos son similares a los encontrados por Morales y Castillo(55); quienes muestran en su estudio que los adolescentes con IRCT en diálisis, deben hacer profundos cambios en su vida cotidiana, debido tanto a la enfermedad como al tratamiento. Muestran además similitudes en lo relacionado con los conflictos de los adolescentes con sus pares sanos, por las demandas de la enfermedad y el tratamiento.

Otro aspecto es el relacionado con el equipo de salud, una revisión(20) que tuvo como objetivo explorar los factores que influyen en la adherencia de los pacientes adultos a la diálisis peritoneal, la medición de la misma y las consecuencias de la no adherencia reportadas en la literatura, reportó la falta de comunicación entre las personas y los profesionales de la salud, 
además encontró en los profesionales de salud, un déficit de herramientas para ayudar a las personas en condición de cronicidad a desarrollar comportamientos adaptativos saludables o modificar los problemáticos; éstos y otros aspectos constituyen serios problemas para el cuidado de sí de los jóvenes con IRCT que están en diálisis.

\section{Discusión}

El propósito del artículo fue realizar un recorrido por algunas bases de datos para conocer los estudios realizados sobre el autocuidado de los adolescentes con IRCT que están en diálisis. En la revisión se evidencia que el autocuidado ha sido abordado principalmente desde el paradigma positivista, con algunas aproximaciones desde el paradigma cualitativo. Desde el enfoque positivista los autores han equiparado el autocuidado con adherencia al tratamiento o también denominada adherencia terapéutica. En esta perspectiva, se ha reconocido que la adherencia a los diferentes tratamientos es baja, y que se requiere intervenciones para mejorarla. Dichas intervenciones deben tener componentes tanto educativos como cognitivo-conductuales.

Los estudios cualitativos por su parte, muestran que las personas con ECNT experimentan diariamente el autocuidado, con sentimientos como el miedo, la tristeza, la incertidumbre, pero también con expectativas y esperanza(55).

Como lo muestra la presente revisión existen múltiples factores que intervienen en la adherencia de los adolescentes a sus regímenes de tratamiento, y en su autocuidado. Tobón(56) menciona que éstos factores son externos e internos, los primeros son de tipo cultural, de género, científicos, tecnológicos, físicos, económicos, políticos y sociales, lo cual hace que el autocuidado constituya un gran desafío para los sistemas, particularmente para el de salud y los internos o personales son los conocimientos, la voluntad, las actitudes y los hábitos. Si bien el abordaje de este fenómeno ha sido multidisciplinario, varios científicos coinciden en que los profesionales de salud tienen el mayor reto, puesto que son los llamados a promoverlo.
Tobón(56) menciona que para el equipo de salud influir en el autocuidado en otras personas requiere un doble desafío, debido a que debe fortalecer su propio autocuidado para tener herramientas para fortalecer el de otros.

Los hallazgos de la revisión indican que capacitar ha sido la principal estrategia utilizada por el personal de salud para fortalecer el autocuidado de las otras personas, ampliando la brecha entre lo que presentan los autores y las necesidades de los jóvenes para realizar su autocuidado. Con las propuestas de los investigadores se puede confirmar que los discursos institucionales que intentan abordar el autocuidado de los adolescentes con ECNT, y particularmente con IRCT, no alcanzan a cubrir las demandas de estos jóvenes puesto que ellos requieren abordajes más integrales que les permitan convivir con su situación de salud de una forma apropiada. En este sentido, es necesario fortalecer la participación del joven en la toma de decisiones y diseño de políticas relacionadas con su salud, pese a que el modelo de cuidado que presenta la OMS establece una conexión entre los sistemas de salud y los jóvenes a través del autocuidado que les permite cierto protagonismo en el sentido de consensuar con el agente de salud situaciones como cambio o flexibilidad en horarios, estrategias para el autocuidado y quizás algunas modificaciones en los lineamientos de las instituciones de salud.

Lo anterior muestra la necesidad de abordajes distintos a los existentes en la literatura publicada para hacer frente a este desafío. Profundizar en cómo estos jóvenes viven diariamente toda la complejidad del autocuidado puede dar herramientas valiosas para entender y fortalecer el fenómeno del autocuidado en esta edad. Particularmente los adolescentes sometidos a diálisis experimentan un deterioro del crecimiento, procedimientos invasivos, limitaciones escolares y sociales. Frecuentemente tienen una baja autoestima y una sensación omnipresente de perder su identidad, integridad corporal, control, independencia y oportunidad(57). Lo anterior sugiere que el cumplimiento del tratamiento renal implica experiencias particulares que son 
difíciles de manejar, que les imponen una gran carga psicosocial y que los hace propensos a padecer alteraciones emocionales.

Es urgente enriquecer el panorama que presenta la literatura científica con las percepciones de los pacientes para conocer cómo viven diariamente su enfermedad y el cuidado de sí mismos, cuáles son sus emociones, desafíos, temores y expectativas. Es crucial escuchar a los adolescentes dadas sus infinitas particularidades, como su incansable lucha por la independencia, que se ve profundamente vulnerada por las imposiciones del sistema de salud para controlar la enfermedad cuando el joven aún no ha desarrollado los recursos y habilidades para afrontar la situación.

En la presente revisión no se recuperaron artículos acerca del autocuidado de adolescentes con IRCT en diálisis, de estudios realizados en el contexto colombiano, no obstante cabe destacar que a estos jóvenes el sistema de salud les presenta un modelo con cobertura adecuada, pero con importantes barreras en el acceso a los servicios de salud(58). En Colombia, hay pocas instituciones de salud que prestan servicios de diálisis y muchos jóvenes tienen que recorrer largas distancias hasta las instituciones con los consiguientes problemas de transporte; los horarios de las instituciones educativas y de los centros de diálisis no les permiten articular la asistencia a la escuela y a la terapia dialítica, afectando profundamente la adherencia a la misma. Los jóvenes también padecen por la inadecuada oportunidad en el acceso a los controles médicos, a la consecución de medicamentos e insumos para la terapia dialítica (este último para el caso de los jóvenes con diálisis peritoneal).

\section{Conclusiones}

Existe un número considerable de investigaciones relacionadas con el autocuidado de adolescentes con ECNT, no obstante hay necesidad de estudios innovadores tanto en los temas que se debe investigar como en el abordaje metodológico, pues la mayoría de los estudios se han centrado en el tratamiento de la enfermedad como el principal elemento constitutivo del autocuidado, específicamente en cuantificar la adherencia al tratamiento farmacológico, dialítico y al impacto de intervenciones educativas destinadas a mejorar dicha adherencia.

Hay carencia de abordajes cualitativos encaminados a conocer el mundo vivencial de las personas, es decir que permitan conocer cómo viven y construyen en su cotidianidad el cuidado estas personas.

La literatura revisada sugiere que existen múltiples factores que interfieren en la adherencia de los adolescentes a sus regímenes de tratamiento, lo cual hace que este aspecto constituya un gran desafío para los sistemas de salud y para los jóvenes mismos. Esto evidencia la necesidad de abordajes distintos a los que presenta la literatura publicada para hacer frente a este desafío tanto en la práctica como en la investigación. Conocer cómo los jóvenes viven diariamente toda la complejidad del autocuidado puede dar herramientas valiosas para entender el fenómeno y fortalecer las intervenciones de apoyo al autocuidado en esta edad tan particular.

\section{Conflictos de interés}

Ninguno declarado.

\section{Referencias}

1. Levin L. Self-care: toward fundamental changes in national strategies. Int J Health Educ. 1981;24:219-28.

2. Godfrey CM, Harrison MB, Lysaght R, Lamb M, Graham ID, Oakley P. Care of self-care by other-care of other: the meaning of self-care from research, practice, policy and industry perspectives. International Journal of Evidence Based Healthcare. 2011;9(1):3-24.

3. Barrera-Ortiz L, Carrillo-González G, Chaparro-Díaz L, Guerrero-Gamboa N, Sánchez-Herrera B. Cuidado al niño en situación de enfermedad crónica. Bogotá: Universidad Nacional de Colombia; 2015. Disponible en: www.editorial.unal.edu.co

4. Collado-Boira EJ. Autocuidados y calidad de vida en pacientes enterostomizados. Moncada (Valencia): Universidad CEU Cardenal Herrera, Facultad de Ciencias de la Salud, Departamento de Fisioterapia; 2014.

5. Sattoe J. Growing up with a Chronic Condition. Instituut Beleid en Management Gezondheidszorg (iBMG), 2014. 
6. Moreno SP, Diaz LC. Reconstruyendo el significado de calidad de vida de los cuidadores en el cuidado: una metasíntesis. Avances en Enfermería 2015;33(1):5566.

7. Organización Mundial de la Salud [Centro de prensa]. Enfermedades no transmisibles [actualidado abril de 2017].

8. Ledón-Llanes L. Enfermedades crónicas y vida cotidiana. Revista Cubana de Salud Pública 2011;37(4):488-499.

9. Figueredo-Borda N. Compañero de vida: enfermedad crónica. Un estudio de caso. Index de Enfermería 2014;23(1-2):46-50.

10. Ministerio de Salud y Protección Social [internet]. ASIS [actualizado 2016].

11. Ministerio de Salud y Protección Social [internet]. Instituto Nacional de Salud. Observatorio Nacional de Salud. Informe 3. Disponible en: https://www.minsalud.gov.co/sites/rid/Lists/.../Infor me3-ME-ONS-definitivo.pdf

12. Roizen M, Figueroa C, Salvia, L. Calidad de vida relacionada con la salud en niños con enfermedades crónicas: comparación de la visión de los niños, sus padres y sus médicos. Archivos Argentinos de Pediatría. 2007;105(4):305-313.

13. Ministerio de Salud y Protección Social [internet]. ASIS [actualizado 2015].

14. Pai AL, Tackett A, Ittenbach RF, Goebel J. Psychosocial Assessment Tool 2.0_General: Validity of a psychosocial risk screener in a pediatric kidney transplant sample. Pediatric Transplantation. 2012;16(1):92-98.

15. Salim TR, Soares GP, Klein CH, Oliveira GMMD. Mortality from Circulatory System Diseases and Malformations in Children in the State of Rio de Janeiro. Brasil: Arquivos Brasileiros de Cardiologia; 2016.

16. Magliano ES, Guedes LG, Coutinho ESF, Bloch KV. Prevalence of arterial hypertension among Brazilian adolescents: systematic review and meta-analysis. BMC Public Health. 2013;13(1):833.

17. Sardinha LB, Santos DA, Silva AM, Grøntved A, Andersen LB, Ekelund U. A comparison between BMI, waist circumference, and waist-to-height ratio for identifying cardio-metabolic risk in children and adolescents. PloS One. 2016;11(2):e0149351.

18. Hooper DK, Williams JC, Carle AC, Amaral S, Chand DH, Ferris ME, Mitsnefes M. The quality of cardiovascular disease care for adolescents with kidney disease: a Midwest Pediatric Nephrology Consortium study. Pediatric Nephrology. 2013;28(6):939-949.

19. Dienemann T, Fujii N, Orlandi P, Nessel L, Furth SL, Hoy WE, et al. International Network of Chronic Kidney Disease cohort studies (iNET-CKD): a global network of chronic kidney disease cohorts. BMC Nephrology. 2016;17:121

20. Carrillo AJ, Marín M, Ortiz L. Adherencia del paciente a la diálisis peritoneal. Repertorio de Medicina y Cirugía. 2014;23(2):112-120.

21. Tong A, Samuel S, Zappitelli M, Dart A, Furth S, Eddy A, Sinha A. Standardised Outcomes in Nephrology-
Children and Adolescents (SONG-Kids): a protocol for establishing a core outcome set for children with chronic kidney disease. Trials. 2016;17(1):40. doi: 10.1186/s13063-016-1528-5

22. Center for Desease Control and Prevention. Chronic Kidney Disease (CKD) Surveillance Project. System United States. Disponible en: http://nccd.cdc.gov/CKD

23. Fernández C, Melgosa M. Enfermedad renal crónica en la infancia. Diagnóstico y tratamiento. Serv. Nefrología Pediátrica. Hosp. Infantil Universitario La Paz. Madrid Protoc diagn ter pediatr. 2014;1:385-401.

24. Orlen S. Aspectos epidemiológicos de insuficiencia renal crónica en los niños en países latinoamericanos. Arch Venez Puericult Pediatr. 2005;131:83-88.

25. Situación de la Enfermedad Renal Crónica, Hipertensión Arterial y Diabetes Mellitus en Colombia 2015. Cuenta de Alto Costo. Bogotá: Fondo Colombiano de Enfermedades de Alto Costo: 2015. Disponible en: www.cuentadealtocosto.com.

26. Goovaerts T, Bagnis-Isnard C, Crepaldi C, Dean J, Melander S, Mooney A, Nilsson EL. Continuing education: preparing patients to choose a renal replacement therapy. Journal of Renal Care. 2015;41(1):62-75.

27. Abreu IS, Kourrouski MFC, Santos DMDSS, Bullinger M, Nascimento, LC, Lima RAGD, Santos CBD. Children and adolescents on hemodialysis: attributes associated with quality of life. Revista da Escola de Enfermagem da USP. 2014;48(4):602-609.

28. Martín LA, Grau JA, Espinosa AD. Marco conceptual para la evaluación y mejora de la adherencia a los tratamientos médicos en enfermedades crónicas. Revista Cubana de Salud Pública. 2014;40(2):222-235.

29. Battersby M, Harris M, Smith D, Reed R, Woodman R. A pragmatic randomized controlled trial of the Flinders Program of chronic condition management in community health care services. Patient Education and Counseling. 2015;98(11):1367-1375.

30. Turner RM, Ma Q, Lorig K, Greenberg J, DeVries AR. Evaluation of a Diabetes Self-Management Program: Claims Analysis on Comorbid Illnesses, Health Care Utilization, and Cost. J Med Internet Res. 2018;20(6).

31. Itla L, Mila U. Fortalecimiento del autocuidado como estrategia de atención primaria de la salud: la contribución de las instituciones de salud en América Latina [Internet]. 2006 [citado 2017 Feb 2]. Disponible en: pwe-hi. bsalud. or/dol/docsonline/get. Php

32. Organización Mundial de la Salud. Adherencia a los tratamientos a largo plazo: pruebas para la acción. Ginebra: OMS; 2004.

33. DiMatteo RM, DiNicola DD. Achieving patient compliance: The psychology of the medical practitioner's role. New York Pergamon Press [Internet]. 1982;5(1):51. Available from: http://deepblue.lib.umich.edu/handle/2027.42/2535 4

34. Bennett D. Compliance in Health Care. Editado por R. Brian Haynes, D. Wayne and David L. Sackett, London: The Johns Hopkins University Press. 1979. Pp 516. Br J Psychiatry. 1980;137(2):190. 
35. MacCarthy S, Saya U, Samba C, Birungi J, Okoboi S, Linnemayr S. "How am I going to live?": exploring barriers to ART adherence among adolescents and young adults living with HIV in Uganda. BMC Public Health. 2018;18:1158

36. Keppeke L, Molina J, Bugni V, de Sande MT, Ascensão L, Dierley G, Schoen TH, Len CA. Psychological characteristics of caregivers of pediatric patients with chronic rheumatic disease in relation to treatment adherence. Pediatric Rheumatology. 2018;16:63

37. Lozano P, Houtrow A. Supporting Self-Management in Children and Adolescents With Complex Chronic Conditions. Pediatrics. 2018;141(suppl 3):S233-S241.

38. Burnier M, Pruijm M, Wuerzner G, Santschi V. Drug adherence in chronic kidney diseases and dialysis. Nephrology Dialysis Transplantation. 2015;30(1):3944.

39. Vasylyeva TL, Singh R, Sheehan C, Chennasamudram SP, Hernández AP. Self-reported adherence to medications in a pediatric renal clinic: psychological aspects. PloS one. 2013;8(7):e69060.

40. Saran R, Bragg-Gresham JL, Rayner HC, Goodkin DA, Keen ML, Van PC, Young E. W. Nonadherence in hemodialysis: associations with mortality, hospitalization, and practice patterns in the DOPPS. Kidney international. 2003;64(1):254-262.

41. Chan KE, Thadhani RI, Maddux FW. Adherence barriers to chronic dialysis in the United States. Journal of the American Society of Nephrology. 2014:25(11):2642-2648. doi: 10.1681/ASN.2013111160

42. Ossareh S, Tabrizian S, Zebarjadi M, Joodat RS. Prevalence of depression in maintenance hemodialysis patients and its correlation with adherence to medications. Iranian Journal of Kidney Diseases. 2014;8(6):467-474.

43. Weisbord SD, Mor MK, Sevick MA, Shields AM, Rollman BL, Palevsky PM, Fine MJ. Associations of depressive symptoms and pain with dialysis adherence, health resource utilization, and mortality in patients receiving chronic hemodialysis. Clinical Journal of the American Society of Nephrology. 2014;9(9):1594-602. doi: 10.2215/CJN.00220114

44. Belayev LY, Mor MK, Sevick MA, Shields AM, Rollman BL, Palevsky PM, Weisbord SD. Longitudinal associations of depressive symptoms and pain with quality of life in patients receiving chronic hemodialysis. Hemodialysis International. 2015;19(2):216-224.

45. Ferris ME, Miles JA, Seamon ML. Adolescents and Young Adults with Chronic or End-Stage Kidney Disease. Blood Purif. 2016;41:205-210.

46. Clark S, Farrington K, Chilcot, J. Nonadherence in dialysis patients: prevalence, measurement, outcome, and psychological determinants. Seminars in dialysis. 2014;27(1):42-49.

47. Cukor D, Ver Halen N, Asher DR, Coplan JD, Weedon J, Wyka KE, Kimmel PL. Psychosocial intervention improves depression, quality of life, and fluid adherence in hemodialysis. Journal of the American Society of Nephrology. 2014;25(1):196-206.
48. Lindsay-Waters A. An ethnography of a children's renal unit: experiences of children and young people with long-term renal illness. Journal of Clinical Nursing. 2008;17(23):3103-3114.

49. Pai AL, McGrady M. Systematic review and metaanalysis of psychological interventions to promote treatment adherence in children, adolescents, and young adults with chronic illness. Journal of Pediatric Psychology. 2014;39(8):918-931.

50. Visekruna S, Edge DS, Keeping-Burke L. "Being in Balance": Self-Management Experiences among Young Women with Type 1 Diabetes. The Qualitative Report. 2015;20(9):1373-1377.

51. Spencer J, Cooper H, Milton B. Qualitative studies of type 1 diabetes in adolescence: a systematic literature review. Pediatric Diabetes. 2010;11(5):364-375.

52. Patrick K, Raab F, Adams M, Dillon L, Zabinski M, Rock C, Norman G. A text message-based intervention for weight loss: randomized controlled trial. Journal of Medical Internet Research. 2009;11(1):e1.

53. Allen C, Vassilev I, Kennedy A, Rogers A. Long-term condition self-management support in online communities: a meta-synthesis of qualitative papers. Journal of Medical Internet Research. 2016;18(3):e61. doi: 10.2196/jmir.5260

54. Wells F, Ritchie D, McPherson AC. 'It is life threatening but I do not mind'. A qualitative study using photo elicitation interviews to explore adolescents' experiences of renal replacement therapies. Child: Care, Health and Development. 2013;39(4):602-612.

55. Morales LC, Castillo E. Vivencias de los (as) adolescentes en diálisis: una vida con múltiples pérdidas, pero con esperanza. Colombia Médica. 2012;38(4):44-53.

56. Tobón 0. El autocuidado una habilidad para vivir. Revista Hacia la promoción de la salud. 2003;8:1-12.

57. Tjaden L, Tong A, Henning P, Groothoff J, Craig JC. Children's experiences of dialysis: a systematic review of qualitative studies. Archives of Disease in Childhood. 2012;9(5):395-402.

58. Molina ME. El modelo de salud colombiano y la calidad del cuidado de enfermería. Revista Aquichán. 2006;6(6):148-155. 